\title{
Radioisotopic assessment of bone metabolism of the operated vertebra after inter-process stabilizer implantation in the lumbar segment of the spine
}

\author{
Maciej Radek ${ }^{1}$, Andrzej Radek ${ }^{1}$, Jacek Rysz ${ }^{2}$, Zbigniew Maziarz ${ }^{3}$, Mariusz Gadzicki \\ Wiesław Tryniszewski ${ }^{3}$
}

\author{
${ }^{1}$ Department of Neurosurgery and Peripheral Nerve Surgery, Medical University \\ of Lodz, Lodz, Poland \\ 2Department of Nephrology, Hypertension and Family Medicine, Medical University \\ of Lodz, Lodz, Poland \\ ${ }^{3}$ Department of Radiological and Isotopic Diagnostics and Therapy, Medical University \\ of Lodz, Lodz, Poland
}

Submitted: 9 December 2014

Accepted: 21 January 2015

Arch Med Sci 2017; 13, 1: 174-182

DOI: 10.5114/aoms.2017.64718

Copyright (C) 2016 Termedia \& Banach

\section{Abstract}

Introduction: Lack of efficacy of pharmacotherapy and physiotherapy in spinal pain syndrome is an indication for intervertebral stabilizer implantation between the processes in the lumbar segment of the spine.

Material and methods: The group consisted of 32 patients qualified after radioisotopic single-photon emission computed tomography/computed tomography (SPECT/CT) examinations with assessment of bone metabolism and mineral density. For comparative purposes, the $L 2$ vertebra was defined as normal. Parameters defined in the area of operated vertebrae were comparable to L2. Imaging examinations and a pain intensity test were performed before and 12 months after the procedure.

Results: In SPECT, osteotropic isotope (OI) activity in spinous bodies and processes was close to $L 2$ values. Density assessed in CT of analysed vertebrae was close to L2. In the control examination, activity of $\mathrm{OI}$ in spinous bodies and processes was higher in the procedure area. Under the stabilizer, there was a strong positive correlation with the $L 2$ parameters. The differences were statistically significant $(p=0.0002)$. The increase of Ol activity in the elements above the stabilizer was variable. In the control examination, there was higher density of spinous processes and bodies above and under the stabilizer. The difference, compared to the L2 density, was statistically significant.

Conclusions: The radioisotopic method with SPECT/CT allows for the precise assessment of bone metabolism in the spine. After the procedure, a negative correlation was observed between bone metabolism changes and pain intensity test results.

Key words: bone diseases, lumbar vertebrae, spondylosis, emissioncomputed tomography.

\section{Introduction}

Different forms of therapy of spinal pain syndrome (SPS) in the lumbar region remain an important issue. The lack of permanent effects of medical therapy is an indication for a neurosurgical procedure [1]. The

\author{
Corresponding author: \\ Wiestaw Tryniszewski MD \\ Department of Radiological \\ and Isotopic Diagnostics \\ and Therapy \\ Medical University of Lodz \\ 113 Zeromskiego St \\ 90-549 Lodz, Poland \\ Phone: +48426393686 \\ E-mail: wieslaw. \\ tryniszewski@umed.lodz.pl
}


modern method of this therapy is the implantation of an inter-process stabilizer between spinal processes in the lumbar segment $[2,3]$. The assessment of bone metabolism is a considerable problem in planning the method of the neurosurgical procedure [4].

For diagnostic and therapeutic purposes, the radioisotopic assessment of bone metabolism changes in the area of the operated vertebra before the procedure and in the distant future provides important information [1]. Image diagnostic methods and the use of a single-photon emission computed tomography/computed tomography (SPECT/CT) hybrid gamma camera allow the functional and morphological bone changes in the spine to be defined. This method allows for the assessment of the influence of the applied surgery on bone metabolism in the site of the performed procedure [4-8]. Complementary tests of pain intensity (VAS linear scale, Oswestry questionnaire) allowed for the assessment of life quality change in the aspect of the performed procedure $[9,10]$. The aim of the study was to assess the influence of an implanted intervertebral stabilizer on the bone metabolism change, measured with SPECT/CT, in the area of the operated vertebrae.

The correlation of imaging examinations with the results of pain intensity and life quality tests was evaluated.

\section{Material and methods}

The examinations were performed in $32 \mathrm{pa}$ tients ( 22 women and 10 men), $54 \pm 5.45$ years of age, treated in the Neurosurgery and Peripheral Nerve Surgery Clinic of the Medical University of Lodz for lumbar spondylosis. The sense of relief at bending forward and the analysis of complementary examinations were the indication for inter-process stabilization in lumbar spondylosis treatment [11].

The results of imaging examinations involving the area of the planned surgical procedure were thoroughly assessed.

Medical documentation imaging stenosis of intervertebral holes at the levels L3/L4 or L4/L5 was analysed. The qualifying examinations for the operated group included computed tomography (CT), magnetic resonance imaging (MRI) and, crucial for achieving the study objective, bone scintigraphy with SPECT/CT and densitometric examination. The observation of pathological intense accumulation foci in the bone system or significant osteoporosis intensification (T-score $<-3.5)$ excluded patients from the analysed group [12-14]. In the discussed group of patients, the stabilizer implantation was performed at the L4/ L5 level in 22 patients and in 10 at the L3/L4 level. Radioisotopic assessment of bone metabolism changes with SPECT/CT in the area of the operated vertebra and the test defining pain intensity were performed twice: before the procedure and 12 months after. These studies were prospective. Due to the fact that there was no comparable group with the same disease state but not treated surgically, morphological and functional parameters of the L2 vertebra were used as the comparative reference point.

\section{Study methods}

1. Anthropometric examinations: age (years), body weight $(\mathrm{kg})$, height $(\mathrm{cm})$, body mass index (BMI; $\mathrm{kg} / \mathrm{m}^{2}$ ).

2. Densitometric examinations: with the NORLAND XR 46 densitometer and dual-energy X-ray absorptiometry (DXA) technique, according to the standard protocol, we defined the bone mineral density (BMD) and T-score of femoral bone neck and lumbar spine in the L2-L5 area. In this study, correct values of BMD and L2 vertebra T-score before and after the surgical procedure were observed.

3. Radioisotopic assessment of bone metabolism. Radioisotopic examination of the bone system was performed with a BrightView XCT hybrid gamma camera $3 \mathrm{~h}$ after the intravenous application of ${ }^{99} \mathrm{TC}-\mathrm{MDP}$ (methyl diphosphate), called an osteotropic isotope (OI), of $11 \mathrm{MBq} / \mathrm{kg}$ c.c. activity. During the first stage, we performed the scintigram of the whole body skeleton with the scan speed $20 \mathrm{~cm} / \mathrm{min}$. On these scintigrams we assessed the normality and quality of Ol accumulation imaging possible metabolic changes. Isotope tomography SPECT of the lumbar segment of the spine was performed at an angle of $360^{\circ}$ with resolution of 20 s/image. Next, in the area of the lumbar spine, CT examination was performed with high resolution $(\mathrm{HR})$ positioning on the vertebrae where the stabilizer was supposed to be implanted. High resolution acquisition was performed with current of $80 \mathrm{~mA}$. In the examinations before and 12 months after the procedure we separately assessed the Ol activity in the spinous body and process. The images obtained in SPECT and CT were analysed simultaneously on two screens of an EBW work station. On the left screen we analysed the images before the procedure and on the right one the images after the procedure (Figure 1).

Possibility of change in bone metabolism of the operated vertebrae was assessed in the images that were a fusion of SPECT and CT.

The morphological changes in processes and the vertebral body were assessed in CT images with isotope resolution of $0.33 \mathrm{~mm}$. In the fusion image the rounded region of interest (ROI) 


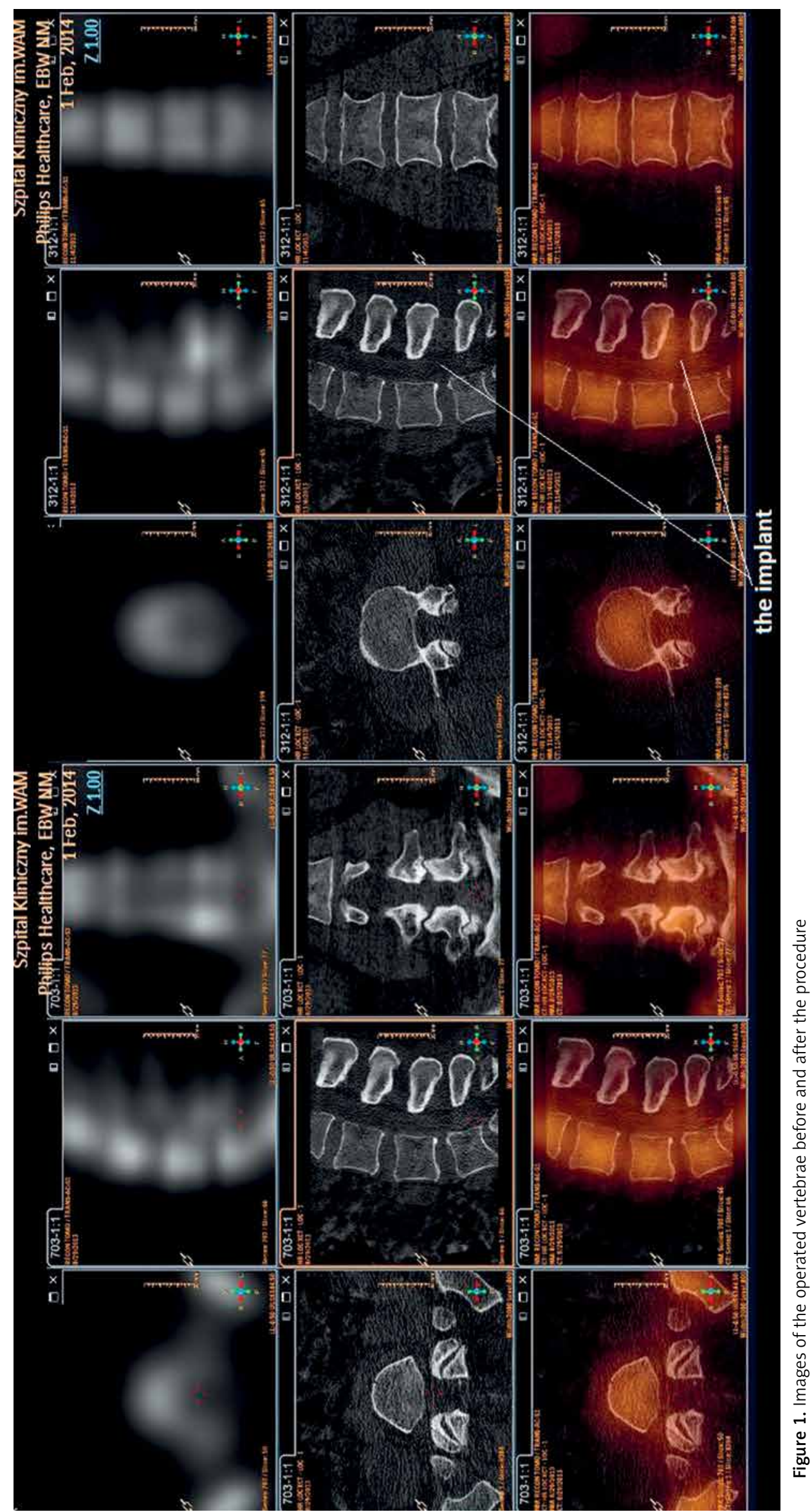


of $100 \mathrm{~mm}^{2}$ was generated. It was then duplicated and placed on processes and vertebral bodies that were close to the stabilizer and on the comparative vertebra L2. The same ROI was copied on the images of the examination performed after the procedure, and it was placed on the same regions that were analysed before the procedure. Next, without changing the position of ROIs, we subtracted the SPECT examination and from ROls we read out the mean value of Hounsfield units (HU). The results obtained in the L2 vertebra were the comparative values for the vertebrae with the stabilizer implanted between. The qualitative assessment of images and quantitative analysis of OI accumulated in spinous bodies and processes allowed us to define bone metabolism before the procedure and 12 months after.

4. Test defining pain characteristics. The measurements of pain intensity before and after the procedure were performed using two scales: visual-analogue scale (VAS) elaborated for graphical and numerical description of pain sensation; and Oswestry questionnaire allowing for the assessment of disability and pain ailments in the spine.

5. Neurosurgical procedure: The patients were operated on in general anaesthesia in the knee-elbow position (Mekki). The location of the operation was defined with fluoroscopy. Next, the typical preparation of the operation field was performed. With a Caspar retractor the access to the spine from one side was performed. After making the hole in the interspinous ligament, we implanted the inter-process stabilizer Orthofix made of polyetheretherketone (PEEK). The implant used belongs to a broad group of socalled interspinous devices. It is not attached permanently to the spinous processes. This is a semi-rigid implant.

The injury was closed in a layered way without leaving drainage. The patients were mobilized the day after the procedure, and after 3 days they left the hospital.

\section{Statistical analysis}

Statistical analysis was performed with the programs Excel 2010 and Statistica 11 PL. The mean value, median and standard deviation were defined. Normal distribution was verified with the Shapiro-Wilk test. In the case of a normal distribution, a classical ANOVA test was used. For measurable variables, the mean value, median, the highest and lowest values and standard deviation were calculated. The test power was separately checked for SPECT and CT. The correlation between the functional and morphological examination results was defined in the same ROIs. The obtained results were compared with the control results obtained in the $\mathrm{L} 2$ vertebra. The calculations were performed using the $95 \% \mathrm{Cl}$.

\section{Results}

\section{Densitometric examinations results}

The results of densitometric examinations performed before the procedure and 12 months after are presented in Table I.

Before the procedure, normal BMD of bones was observed in 14 patients (44\% of the group), and in 18 patients (56\%) we observed slight osteopenia. Similar results were obtained 12 months after the procedure. The BMD of the analysed vertebrae (L2-L5) 12 months after the procedure was comparable with previous results, and the observed differences were not statistically significant.

\section{SPECT/CT scintigraphy}

To make the result presentation simpler and for the purposes of this study we adopted the following symbols: I - process under stabilizer, II - vertebral body under stabilizer, III - process above stabilizer, IV - vertebral body above stabilizer, $\mathrm{V}$ - process of vertebra L2; $\mathrm{VI}$ - body of vertebra $\mathrm{L} 2$.

The values of $\mathrm{Ol}$ activity accumulated in spinous bodies and processes in particular examinations before the procedure are presented in Figure 2.

Table I. Results of densitometric examinations $(N=32)$

\begin{tabular}{|c|c|c|c|c|c|c|}
\hline \multirow[t]{2}{*}{ Parameter } & \multicolumn{2}{|c|}{ Spine L2 } & \multicolumn{2}{|c|}{ Spine L3-L5 } & \multicolumn{2}{|c|}{ Femoral neck } \\
\hline & BMD $\left[\mathrm{g} / \mathrm{cm}^{2}\right]$ & $T$-score & $\mathrm{BMD}\left[\mathrm{g} / \mathrm{cm}^{2}\right]$ & $T$-score & $\mathrm{BMD}\left[\mathrm{g} / \mathrm{cm}^{2}\right]$ & $T$-score \\
\hline \multicolumn{7}{|c|}{ Examinations before the procedure: } \\
\hline Mean & 1.1761 & 0.1735 & 1.1001 & -0.0859 & 0.9089 & -0.8153 \\
\hline Standard deviation & 0.0897 & 0.1082 & 0.1290 & 0.7096 & 0.0717 & 0.6385 \\
\hline \multicolumn{7}{|c|}{ Examinations after the procedure: } \\
\hline Mean & 1.1998 & 0.1528 & 1.1572 & -0.3350 & 0.9001 & -0.8900 \\
\hline Standard deviation & 0.0811 & 0.1156 & 0.1343 & 0.9131 & 0.0762 & 0.6659 \\
\hline
\end{tabular}


Scatter plot with histograms of I against $\mathrm{V}$

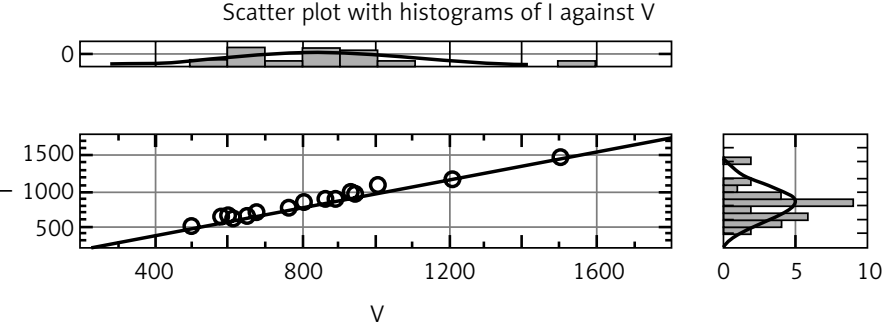

$\mathrm{V}: N=32$, mean $=842.5313, \mathrm{SD}=251.2559, \max .=1508, \min .=500$

$\mathrm{I}: N=32$, mean $=824.2697, \mathrm{SD}=251.2571, \max .=1488, \min .=459$

$\mathrm{V}: \mathrm{I}-r=0.9951, p<0.0001$

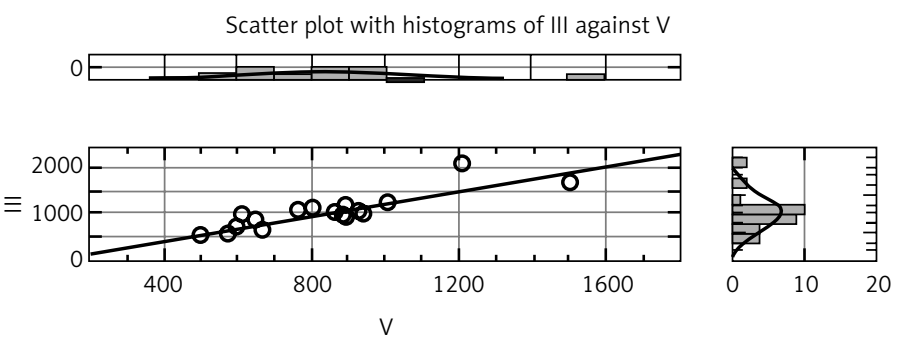

$\mathrm{V}: N=32$, mean $=842.5313, \mathrm{SD}=251.2559, \max .=1508, \min .=500$

III: $N=32$, mean $=1005.8345, \mathrm{SD}=376.7373, \max .=2006, \min .=486$

$\mathrm{V}:$ III $-r=0.8794, p<0.0001$

Scatter plot with histograms of II against VI

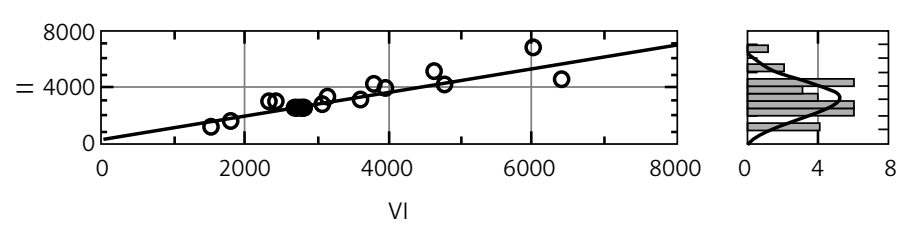

VI: $N=32$, mean $=3451.5791, \mathrm{SD}=1294.1871, \max .=6409, \min .=1555$

II: $N=32$, mean $=3128.0931, \mathrm{SD}=1237.6707, \max .=6800, \min .=1179$

$\mathrm{VI}: \mathrm{II}-r=0.8833, p<0.0001$

Scatter plot with histograms of IV against $\mathrm{V}$

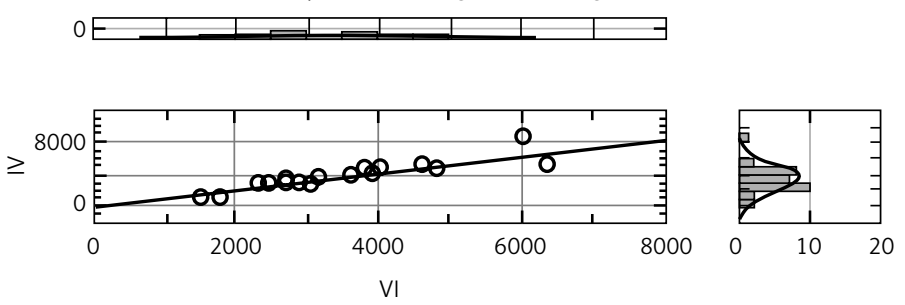

VI: $N=32$, mean $=3451.5791, S D=1294.1871, \max .=6409, \min .=1555$

IV: $N=32$, mean $=3541.125, \mathrm{SD}=1521.6527, \max .=8345$, min. $=867$

$\mathrm{VI}:$ IV $-r=0.8836, p<0.0001$

Figure 2. Values of accumulated $\mathrm{Ol}$ activity in operated vertebra and correlation of the obtained results with OI values in $\mathrm{L} 2$ vertebra before the procedure

Values of Ol activity accumulated in processes and vertebral bodies of the operated vertebrae after the procedure are presented in Figure 3.

In the examination 12 months after the procedure, Ol activity in processes and vertebral bodies was increased in the operated area. The analysed parameters in the vertebral body and process un- der the stabilizer showed a strong positive correlation with the parameters of vertebra L2. Yet, in the vertebral body and process above the stabilizer, the $\mathrm{OI}$ activity was variable, and the correlation with the parameters of $\mathrm{L} 2$ was positive but moderate. 


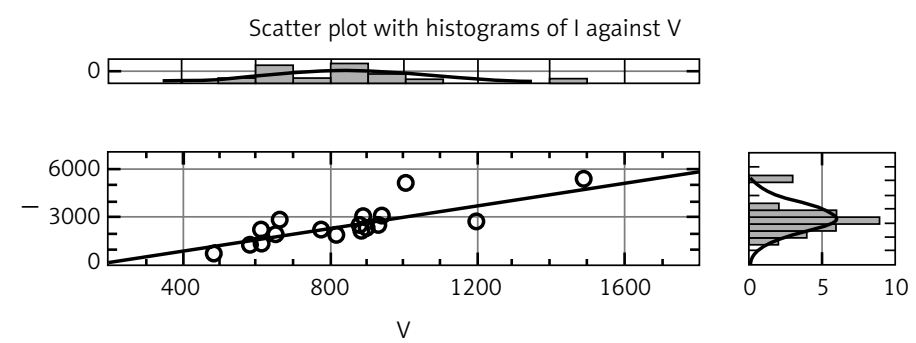

$\mathrm{V}: N=32$, mean $=844, \mathrm{SD}=248.0865, \max .=1500, \min .=498$

$\mathrm{I}: N=32$, mean $=2382.25, \mathrm{SD}=1075.3931, \max .=5171, \mathrm{~min} .=756$

$$
\mathrm{V}: \mathrm{I}-\mathrm{r}=0.8276, p<0.00001
$$

Scatter plot with histograms of III against V
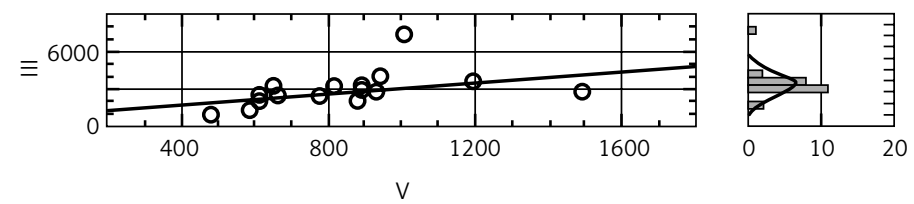

$\mathrm{V}: N=32$, mean $=844, \mathrm{SD}=248.0865, \max .=1500, \min .=498$

III: $N=32$, mean $=2669.8152, \mathrm{SD}=1085.9786, \max .=7038, \min .=851$

$\mathrm{V}: \mathrm{III}-r=0.5082, p=0.0030$

Scatter plot with histograms of II against VI
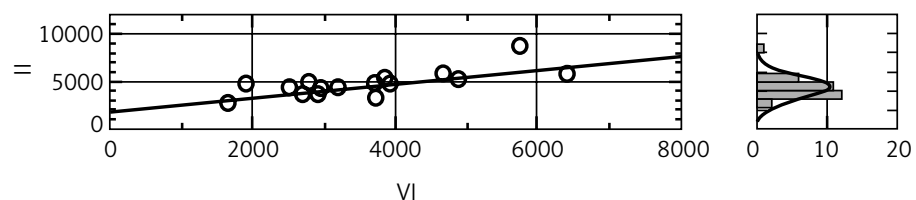

$\mathrm{VI}: N=32$, mean $=3503.9469, \mathrm{SD}=1246.1507, \max .=6435, \min .=1648.38$ II: $N=32$, mean $=4322.1275, \mathrm{SD}=1209.5343, \max .=8731, \min .=2340$ $\mathrm{VI}: \| \mathrm{I}-r=0.7547, p<0.00001$

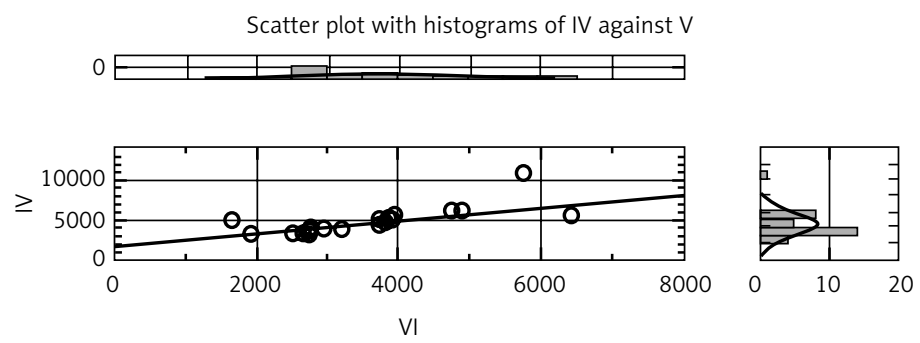

$\mathrm{VI}: N=32$, mean $=3503.9469, \mathrm{SD}=1246.1507, \max .=6435, \min .=1648.38$ IV: $N=32$, mean $=4389.3125, \mathrm{SD}=1533.0393, \max .=10673, \min .=2870$ $\mathrm{VI}: \mathrm{IV}-r=0.6585, p=0.00004$

Figure 3. Values of $\mathrm{Ol}$ activity accumulated in operated vertebrae and correlation of the obtained results with $\mathrm{OI}$ values in $\mathrm{L} 2$ vertebra after the procedure

\section{$\mathrm{CT}$ examinations}

In CT examination, analogically to scintigraphic examination, as the comparative value we took the density of the process and the body of the $L 2$ vertebra expressed in $\mathrm{HU}$ units.

A very strong correlation of the density of the process and the body of L2 was observed when comparing before and 12 months after the procedure.

Before the procedure the density in vertebral bodies and processes under the stabilizer was close to the density of L2 elements, and the correlation between them was strong and positive $(r>0.9)$. Density in vertebrae above the stabilizer 
was heterogeneous. The correlation with density values of the L2 process was positive and weak ( $r=0.4693)$, and with the L2 body it was positive and moderate $(r=0.6393)$.

In the examination 12 months after the procedure the density in vertebral bodies and processes under and above the stabilizer was increased compared to the examinations before the procedure. The observed difference, compared to the density in L2, was statistically significant. In all operated patients the increase of this value in vertebrae under the stabilizer was comparable. In CT, analogically to SPECT, the density increase in the examined elements above the stabilizer was variable. The statistical dependency between SPECT and CT was also assessed in the area of the operated vertebrae. Before the procedure, the correlation SECT vs. CT in the area of processes under the stabilizer was positive and strong, and it was positive and weak in the area of vertebral bodies of these vertebrae. We did not observe any statistical dependency between SPECT and CT in vertebral bodies and processes above the stabilizer. Analogical associations were observed after the surgery.

The obtained results were verified with a test. We adopted a two-sided null hypothesis with the assumption of type I error probability $\alpha=0.05$. For the comparable value we assumed the parameters obtained in the $L 2$ vertebra. The results of the test power in SPECT and CT examinations 12 months after the procedure are presented in Table II.

The results of the tests defining pain characteristics and life quality before and after the surgery are presented in Table III.

The results of pain intensity measurement on the VAS scale before vs after the procedure showed a strong positive correlation $(p=0.00002)$. The as- sessment with the Oswestry questionnaire had weaker power $(r=0.6843$ and $p=0.0024)$. Before the procedure we did not observe statistical dependency between the results of the Oswestry questionnaire and the quantity of $\mathrm{Ol}$ accumulated in processes. However, 12 months after the procedure, the correlation was negative and moderate $(r=-0.4653)$.

\section{Discussion}

Along with the lifespan prolongation, more and more people suffer from pain of the lumbar segment of the spine $[15,16]$. If conservative treatment does not bring required effects, spine surgery is the recommended option. First implantation of metal implants within the spinous process was associated with their frequent falling out, which limited the development of this technique [17]. New-generation inter-process stabilizers are often used in surgical treatment of the spinal lumbar segment $[2,18,19]$. Appropriate selection of the deformation correction technique allows for significant reduction of pain and the amelioration of life quality and physical efficiency of the patient [20-22]. Proper pre-operative planning of the surgery requires precise, multi-methodological use of imaging examination in assessment of the bone system [23]. This is also confirmed by the results of the present study. However, there is a risk of possible complications such as the spinous processes' fracture or collapse $[11,16]$. We did not observe such complications in our study. Our study contributes to the studies of clinical effectiveness of the In-Swing implant and the assessment of how this method of treatment influences spinal bone metabolism within operated vertebrae. Methods

Table II. Power of test of SPECT and CT examinations 12 months after surgery

\begin{tabular}{|c|c|c|c|c|c|c|c|}
\hline \multicolumn{8}{|c|}{ Power of SPECT and CT; one mean; test $t ; \mathrm{HO}: \mathrm{Mi}=\mathrm{MiO}$} \\
\hline \multicolumn{4}{|c|}{ SPECT of vertebra } & \multicolumn{4}{|c|}{ CT of vertebra } \\
\hline \multicolumn{2}{|c|}{ Under the stabilizer } & \multicolumn{2}{|c|}{ Over the stabilizer } & \multicolumn{2}{|c|}{ Under the stabilizer } & \multicolumn{2}{|c|}{ Over the stabilizer } \\
\hline $\begin{array}{l}\text { Spinous } \\
\text { process }\end{array}$ & $\begin{array}{c}\text { Vertebral } \\
\text { body }\end{array}$ & $\begin{array}{l}\text { Spinous } \\
\text { process }\end{array}$ & $\begin{array}{c}\text { Vertebral } \\
\text { body }\end{array}$ & $\begin{array}{l}\text { Spinous } \\
\text { process }\end{array}$ & $\begin{array}{c}\text { Vertebral } \\
\text { body }\end{array}$ & $\begin{array}{l}\text { Spinous } \\
\text { process }\end{array}$ & $\begin{array}{c}\text { Vertebral } \\
\text { body }\end{array}$ \\
\hline 0.7960 & 0.7974 & 0.7187 & 0.8791 & 0.7766 & 0.8098 & 0.7911 & 0.8831 \\
\hline
\end{tabular}

Table III. Assessment of pain before and after the procedure with the pain scale $(N=32)$

\begin{tabular}{|lcccc|}
\hline Parameter & \multicolumn{2}{c}{ VAS scale } & & \multicolumn{2}{c|}{ Oswestry questionnaire } \\
\cline { 2 - 5 } & Before & After & Before & After \\
\hline Mean & 76.47 & 29.71 & 30.06 & 11.88 \\
\hline Standard deviation & 6.68 & 5.09 & 2.66 & 1.68 \\
\hline Min. & 60.00 & 20.00 & 25.00 & 8.00 \\
\hline Max. & 76.47 & 29.71 & 30.06 & 29.88 \\
\hline
\end{tabular}


of imaging diagnostics are rapidly evolving, and it seems that examination of the bone system with $\mathrm{MRI}$ is not always sufficient. Bone scintigraphy allows for the exclusion of other disease states resulting in spine pain symptoms. Radioisotopic methods (SPECT/CT) give a unique possibility for the assessment of metabolic activity in the bone system [23].

Radioisotopic (SPECT/CT) examinations of vertebrae before and after the procedure of intervertebral stabilizer implantation allow for quantitative assessment of local bone metabolism changes. This, in turn, enables accurate qualification for the planned procedure and the assessment of remote effects of the therapy. The observed great power of the test confirms that the obtained results are reliable in the assessment of bone metabolism changes in the area of operated vertebrae. The advancement of CT technologies in the evaluation of bone quality makes it a suitable tool for use in clinical diagnostics. The HU values obtained in CT correlate with the bone mineral density. Computed tomography examination allows for morphological assessment of the spinal processes and vertebral body, which along with SPECT enable the assessment of bone metabolism [24]. The novelty of this study involves the broadening of radiological methods with radioisotopic examinations, mainly bone scintigraphy performed with SPECT/ $\mathrm{CT}$ technique to obtain full information on the morphological and functional state of the bone system. The SPECT is a functional examination in which the level of $\mathrm{Ol}$ accumulation is directly proportional to metabolic processes in the bone system. The utilization of scintigraphy, which is sensitive but has low specificity, in combination with computed tomography having high specificity but low sensitivity is most useful in such cases [25]. Before the procedure, the Ol activity in vertebral bodies and spinous processes in the area of the implemented stabilizer showed a strong positive correlation with the activity in comparative L2 vertebra, and the observed differences were not statistically significant. This means that the bone metabolism in all analysed vertebrae before the procedure was similar. The analysis of Ol activity in the vertebral body and spinous process of comparative vertebra L2 before and after the procedure did not show any statistically significant differences. This confirms that the stabilizer implantation did not influence metabolic changes in other vertebrae. Increased accumulation of $\mathrm{OI}$ activity in SPECT and raised density in CT examination of the operated vertebra observed after 12 months were the confirmation of increased bone metabolism. Enhanced bone metabolism could be a result of heterogeneous vertebral kinetics and load transfer in response to the implant.
It could also be a result of both the elimination of friction among vertebrae and the direct impact of the stabilizer on adjacent spinous processes [15, 26]. Increased bone metabolism could also be due to surgery-associated enlargement of the space between vertebrae. It also enabled additional nourishment of the intervertebral disc and thereby the improvement of perfusion within the outer vertebral areas [16]. Following the stabilizer implantation and the decompression of vertebrae, spinous processes took over the function of keeping the proper distance between the vertebrae. One of the advantages of radioisotopic examination is the possibility to assess the rate of bone restructuring processes even in small regions of interest (ROIs) [6]. Standard SPECT examination does not allow for full quantity assessment of regional accumulation of osteotropic isotope activity [4-6]. Quantitative evaluation of bone metabolism is possible with the use of SPECT/ CT technique [5-7]. The obtained results demonstrate that the radioisotopic method of spine examination SPECT/CT with ${ }^{99 m}$ Tc-MDP is helpful for defining bone metabolism and therapy effects. This study demonstrated that the disburdening of the operated spine segment was associated with an increase in bone metabolism expressed in the form of increased density of mainly spinous processes and to a lesser degree of vertebral bodies. The effects of therapy following intervertebral stabilizer implantation were better than in the patients treated noninvasively and improvement of patients' quality of life was also observed.

A year after the surgery, all the patients reported a decrease of pain intensity assessed with the use of the VAS scale. In case of the Oswestry questionnaire, 23 patients declared evident improvement, 6 patients reported incomplete improvement, and 3 patients lack of improvement in comparison to the condition before the surgery. The results suggest that the implant may provide a more effective treatment than other surgical procedures $[1,27]$. The use of a stabilizer is safe and efficient and less invasive in comparison to the opening of the spinal canal, and it also cures lumbar spinal stenosis [28]. The applied method provided important information concerning bone metabolism in case of a less invasive method of spine surgery [4].

Decompression of the intervertebral space obtained as a result of stabilizer implantation resulted in reduced pain and improved quality of patients' life.

This was reflected by the smaller power of correlation of the obtained results before and after the procedure $(r=0.6843)$, while with the VAS pain scale the correlation was $r=0.8423$.

In conclusion, the radioisotopic method with SPECT/CT technique allows for high thoroughness in bone metabolism assessment. Stabilizer 
implantation causes a bone metabolism increase after 12 months in the area of operated vertebrae, which is expressed by increased mineralization and density mainly in the area of vertebral processes. After the procedure we observed a negative correlation between bone metabolic changes and the results of pain intensity tests.

\section{Acknowledgments}

The study was performed within the investigations and statutory activity of the Department of Diagnostics and Radiological and Isotopic Therapy, Medical University, Lodz.

\section{Conflict of interest}

The authors declare no conflict of interest.

\section{References}

1. Hsu KY, Zucherman JF, Hartjen CA, et al. Quality of life of lumbar stenosis-treated patients in whom the X STOP interspinous device was implanted. J Neurosurg Spine 2006; 5: 500-7.

2. Kubaszewski Ł, Nowakowski A, Gasik R, Łabędź W. Intraobserver and interobserver reproducibility of the novel transcription method for selection of potential nerve root compression in MRI study in degenerative disease of the lumbar spine. Med Sci Monit 2013; 19: 216-21.

3. Schilling C, Pfeiffer M, Grupp TM, Blömer W, Rohlmann A. The effect of design parameters of interspinous implants on kinematics and load bearing: an in vitro study. Eur Spine J 2014; 23: 762-71.

4. Sandu N, Schaller B. Spinal molecular imaging by (68) Ga-DOTATATE-positron emission tomography. J Craniovertebr Junction Spine 2014; 5: 139-40.

5. Tryniszewski W, Gadzicki M, Gorska-Chrzastek M, Rysz J, Maziarz Z. Bone metabolism assessment, bone metabolism index designation and the determination of its normal values range in young healthy women. Med Sci Monit 2011; 17: CR563-71.

6. Blake GM, Moore AE, Fogelman I. Quantitative studies of bone using $(99 \mathrm{~m}) \mathrm{Tc}$-methylene diphosphonate skeletal plasma clearance. Semin Nucl Med 2009; 39: 369-79.

7. Ergün EL, Kiratli PO, Günay EC, Erbaş B. A report on the incidence of intestinal $99 \mathrm{mTc}$-methylene diphosphonate uptake of bone scans and a review of the literature. Nucl Med Commun 2006; 27: 877-85.

8. Lehman VT, Murphy RC, Maus TP. 99mTc-MDP SPECT/ $C T$ of the spine and sacrum at a multispecialty institution: clinical use, findings, and impact on patient management. Nucl Med Commun 2013; 34: 1097-106.

9. Fritz JM, Irrgang JJ. A comparison of a modified Oswestry Low Back Pain Disability Questionnaire and the Quebec Back Pain Disability Scale. Phys Ther 2001; 81: 776-88.

10. Fairbank JC, Pynsent PB. The Oswestry Disability Index. Spine 2000; 25: 2940-52.

11. Willén J, Danielson B, Gaulitz A, Niklason T, Schönström N, Hansson T. Dynamic effects on the lumbar spinal canal: axially loaded CT-myelography and MRI in patients with sciatica and/or neurogenic claudication. Spine 1997; 22: 2968-76.

12. Bonaldi G, Bertolini G, Marrocu A, Cianfoni A. Posterior vertebral arch cement augmentation (spinoplasty) to prevent fracture of spinous processes after interspinous spacer implant. AJNR Am J Neuroradiol 2012; 33: 522-8.

13. Kallmes DF, Comstock BA, Heagerty PJ, et al. A randomized trial of vertebroplasty for osteoporotic spinal fractures. N Engl J Med 2009; 361: 569-79.

14. Buchbinder R, Osborne RH, Ebeling PR, et al. A randomized trial of vertebroplasty for painful osteoporotic vertebral fractures. N Engl J Med 2009; 361: 557-68.

15. Lindsey DP, Swanson KE, Fuchs P, et al. The effects of an interspinous implant on the kinematics of the instrumented and adjacent levels in the lumbar spine. Spine 2003; 28: 2192-7.

16. Kim DH, Shanti N, Tantorski ME, et al. Association between degenerative spondylolisthesis and spinous process fracture after interspinous process spacer surgery. Spine I 2012; 12: 466-72.

17. Nielsen M. X-STOP surgical implant for the treatment of lumbar spinal stenosis: clinical practice recommendations for neurosurgical nurse practitioners. J Neurosci Nurs 2013; 45: 44-51.

18. Chiu JC. Interspinous process decompression (IPD) sys tem (X-STOP) for the treatment of lumbar spinal stenosis. Surg Technol Int 2006; 15: 265-75.

19. Knowles FL. The knowles vertebral support operation J lowa State Med Soc 1958; 48: 551-4.

20. Sénégas J, Vital JM, Pointillart V, Mangione P. Clinical evaluation of a lumbar interspinous dynamic stabilization device (the Wallis system) with a 13-year mean follow-up. Neurosurg Rev 2009; 32: 335-41.

21. Scuderi GJ, Brusovanik GV, Fitzhenry LN, Vaccaro AR. Is wound drainage necessary after lumbar spinal fusion surgery? Med Sci Monit 2005; 11: CR64-6.

22. Fraser JF, Huang RC, Girardi FP, Cammisa FP Jr. Pathogenesis, presentation, and treatment of lumbar spinal stenosis associated with coronal or sagittal spinal deformities. Neurosurg Focus 2003; 14: e6.

23. Sandu N, Schaller B. Commentary. J Neurosci Rural Pract 2014; 5: 159-60.

24. Lee S, Chung CK, Oh SH, Park SB. Correlation between bone mineral density measured by dual-energy $\mathrm{x}$-ray absorptiometry and Hounsfield units measured by diagnostic CT in lumbar spine. J Korean Neurosurg Soc 2013; 54: 384-9.

25. Scheyerer M, Pietsch C, Zimmermann S, Osterhoff G, Simmen $\mathrm{H}$, Werner CM. SPECT/CT for imaging of the spine and pelvis in clinical routine: a physician's perspective of the adoption of SPECT/CT in a clinical setting with a focus on trauma surgery. Eur I Nucl Med Mol Imaging 2014; 41 Suppl 1: S59-66.

26. Zhao X, Wu ZX, Han BJ, Yan YB, Zhang Y, Lei W. Three dimensional analysis of cervical spine segmental motion in rotation. Arch Med Sci 2013; 9: 515-20.

27. Karabekir HS, Yildizhan A, Atar EK, Yaycioglu S, Gocmen-Mas N, Yazici C. Effect of ligamenta flava hypertrophy on lumbar disc herniation with contralateral symptoms and signs: a clinical and morphometric study. Arch Med Sci 2010; 6: 617-22.

28. Siddiqui $M$, Smith FW, Wardlaw D. One-year results of $X$ Stop interspinous implant for the treatment of lumbar spinal stenosis. Spine (Phila Pa 1976) 2007; 32: 1345-8. 